\title{
Invited Commentary: Childhood and Adolescent Obesity: Psychological and Behavioral Issues in Weight Loss Treatment
}

\author{
David B. Sarwer • Rebecca J. Dilks
}

Received: 5 May 2011/Accepted: 11 May 2011/Published online: 31 May 2011

(C) Springer Science+Business Media, LLC 2011

\begin{abstract}
The prevalence of childhood and adolescent obesity has tripled in the past three decades. This increase has been accompanied by a dramatic rise in obesity-related health complications among American youth. Thus, many obese youth are now experiencing illnesses that will threaten their life expectancy in the absence of significant weight loss. Despite these concerns, a relatively modest body of research has focused on the treatment of adolescent obesity. Results from trials investigating the efficacy of behavioral and pharmacological treatments, like studies of these interventions with adults, suggest that individuals typically lose 5-10\% of their initial weight. Unfortunately, weight regain is common. Given the increase in the number of obese adolescents, coupled with the modest results from more conservative treatment approaches, it is not surprising that bariatric surgery for adolescents who suffer from extreme obesity has grown in popularity. The weight losses after surgery are impressive and many adolescents, like adults, experience significant improvements in their physical and mental health postoperatively. However, only a small fraction of adolescents and adults who are heavy enough for bariatric surgery present for surgical treatment. Among those who undergo surgery, a significant minority appear to struggle with a number of behavioral and psychosocial issues that threaten their lifelong success. With all of this in mind, the current obesity problem in the United States and other Westernized countries likely will present a significant challenge to both current and future
\end{abstract}

D. B. Sarwer $(\bowtie) \cdot$ R. J. Dilks

Center for Weight and Eating Disorders,

University of Pennsylvania School of Medicine,

3535 Market St., Suite 3121, Philadelphia, PA 19104-3309, USA

e-mail: dsarwer@mail.med.upenn.edu medical and mental health professionals who work with adolescents and young adults.

\section{The Childhood and Adolescent Obesity Problem}

Obesity is a growing problem among America's youth. The rate of obesity or overweight ( $>95$ th percentile for age and gender) has doubled among children and tripled among adolescents over the last 20 years (Ogden et al. 2002). The most recent data suggests that $31 \%$ of children in the United States are currently overweight or obese (Ogden et al. 2010), which translates into approximately 5 million children. Furthermore, recent estimates suggest that $4 \%$ of American children and adolescents are above the 99th percentile and, thus, are extremely obese (Freedman et al. 2007). This percentage is larger than the number of American youth affected by cancer, cystic fibrosis, HIV and type I diabetes mellitus combined (Freedman et al. 2007).

Instead of using the term "obesity" with children and adolescents, several authorities recommend using the Centers for Disease Control's (CDC) BMI tables (Kuczmarski et al. 2002). The CDC guidelines do not label individuals as "obese". Instead, "at risk of overweight" is used to describe youth between the 85th and 94th BMI percentile and "overweight" is used for those above the 95th percentile (Dietz and Bellizzi 1999; Kuczmarski et al. 2002). By later adolescence, the 95 th percentile approaches the definition of adult obesity, $\approx 30 \mathrm{~kg} / \mathrm{m}^{2}$ (Cole et al. 2000). Generally, the term "obesity" is only used for the problem at the population level and for individual children when adiposity has been confirmed by another measure in addition to BMI (Speiser et al. 2005).

A particularly alarming trend suggests that the heaviest youth are becoming heavier, thus placing more individuals 
at greater risk for more immediate and serious weightrelated health problems. Overweight and obesity are associated with insulin resistance, type II diabetes mellitus, cardiovascular risk, menstrual irregularities, obstructive sleep apnea, non-alcoholic fatty liver disease, and psychosocial problems in children and adolescents (Daniels et al. 2005; Zeller et al. 2006). Adolescent obesity is known to increase the rate of atherosclerosis, type II diabetes mellitus, coronary heart disease, hip fractures, and gout in adulthood. An investigation from the Nurses' Health Study found that obese adolescents had an almost 3-fold increase in premature deaths (van Dam et al. 2006). The social impact of obesity, especially on females, is staggering. As obese female adolescents age, they are likely to suffer extreme social consequences from obesity, including achieving less educational status, earning less money, being more likely to live in poverty, and being less likely to marry (Gortmaker et al. 1993).

These physical and psychological issues recently have led researchers to begin to study the efficacy of neighborhood and school-based obesity prevention programs (e.g., Foster et al. 2008). The goal of these programs is to increase physical activity, decrease sedentary behavior and/ or reduce caloric intake. These approaches involve policies to alter the social, regulatory or physical environments of children and adolescents to promote the engagement of healthy behaviors, even if the child is not aware that they are adopting these behaviors (Robinson and Sirard 2005). By starting early, the goal is to avoid excess weight gain as the children go through adolescence and into adulthood (Caballero 2004).

Although these programs may be shown to have some impact, it is unlikely that these will stop, let alone reverse, the obesity epidemic (Boutelle et al. 2011). Thus, most researchers and clinicians remain focused on the issue of treatment. Below, we will provide a review of the treatment of childhood and adolescent obesity. We begin with an overview of more conservative treatment approaches, such as behavioral modification and pharmacotherapy. The limited efficacy and durability of these approaches has led to interest in the surgical treatment of obese adolescents. Thus, the commentary concludes with a discussion of recent and ongoing research on the use of bariatric surgery for obese adolescents.

\section{Behavioral Modification and Pharmacotherapy for Adolescent Obesity}

The treatment of childhood and adolescent obesity, as compared to adult obesity, has been sadly neglected. Fewer than a dozen controlled trials have been conducted with adolescents. Most have examined lifestyle modification programs that included dietary and exercise counseling, behavioral modification strategies, and, in some studies, parental participation. This approach is effective at producing moderate weight losses of $2-4 \mathrm{~kg}$, with many adolescents remaining substantially obese (BMI $\geq 35 \mathrm{~kg} / \mathrm{m}^{2}$ ) at the end of treatment (e.g. Robinson 1999; Johnson et al. 1997).

Within the past decade, investigators have tried a number of strategies to maximize initial weight loss and promote more successful long term maintenance (e.g. Berkowitz et al. 2003, 2006, 2010; Oude Luttikhuis et al. 2009). These approaches have typically involved the combination of behavioral treatment (BT) and either pharmacotherapy or the use of meal-replacement products designed to reduce portion sizes and control total caloric intake. For example, Berkowitz et al. (2003) randomized 82 adolescents to BT with either placebo or sibutramine, a medication designed to promote feelings of satiety. As is often done in behavioral based treatment for weight loss, adolescents in both conditions were instructed to consume a self-selected diet of approximately $1300 \mathrm{kcal} / \mathrm{d}$ and were encouraged to engage in $\geq 120 \mathrm{~min}$ per week of aerobic exercise. At months 3 and 6, adolescents treated by BT+ sibutramine lost more than twice as much weight as those treated by BT + placebo ( $7.8 \mathrm{~kg}$ vs. $3.2 \mathrm{~kg} ; p<.001)$. The $\mathrm{BT}+$ sibutramine group had an $8.5 \pm 6.8 \%$ reduction in initial BMI compared to a $4.0 \pm 5.4 \%$ loss in the BT+ placebo group $(p<.001)$, suggesting that the combination of BT and pharmacotherapy can be used to promote greater weight loss.

Although these results were encouraging, the Food and Drug Administration removed sibutramine from the United States market in 2010 because of concerns about an association with heart disease. At present there is only one medication, orlistat, approved for long-term usage in the United States. However, as it is rarely covered by insurance, its usage by pediatricians to treat adolescents is quite infrequent.

Furthermore, there are a number of additional limitations to both behavioral and pharmacologically based treatments. First, while modest weight losses of 5-8\% of initial body weight may improve the health and psychosocial status of those with moderate obesity, they may have little effect on the health and well being of the extremely obese. Second, the majority of obese adults treated with behavioral and pharmacologic treatments typically regain weight over time. Little is known about successful, long term weight maintenance in obese adolescents.

For these and other reasons, investigators have turned their attention to bariatric surgery as a treatment for adolescents with extreme obesity who may have tried (and failed) these more conservative treatments or for whom the severity of their obesity is so great that the more modest 
weight losses of these treatments are likely to have little impact on their physical health and psychosocial status. While this may sound preposterous to some, there is compelling evidence to suggest that obese children and adolescents are likely to become obese adults and, as a result, be at risk for premature death (Whitaker et al. 1997). This observation, coupled with the medical and psychosocial toll that obesity can take on an obese adolescent (as noted above), provides further support for the need for more aggressive interventions.

\section{Surgical Treatment of Obesity}

Bariatric surgery is the most effective weight control option for obesity. Bariatric surgery is currently recommended for individuals 18 years and older with a BMI $\geq 40 \mathrm{~kg} / \mathrm{m}^{2}$ (or a BMI $>35 \mathrm{~kg} / \mathrm{m}^{2}$ in the presence of significant co-morbidities) (Consensus Development Conference Panel 1991). The most reliable statistics published to date suggest that 103,000 individuals underwent bariatric surgery in the United States in 2003 (Santry et al. 2005); more recent estimates suggest that over 200,000 procedures are performed annually. Approximately 3,000 bariatric surgical procedures were performed on adolescents between 1996-2003, with a 3-fold increase between 2000 and 2003 (Tsai et al. 2007). These numbers are predicted to increase in the future. However, relatively little is known about the safety and efficacy of bariatric surgery in adolescents.

The patient selection criteria for adults interested in bariatric surgery are well established. An American Academy of Pediatrics expert panel developed guidelines for considering bariatric surgery in adolescents (Inge et al. 2004b). In brief, the panel recommended surgery for adolescents with BMI $\geq 50 \mathrm{~kg} / \mathrm{m}^{2}$ or BMI $\geq 40 \mathrm{~kg} / \mathrm{m}^{2}$ in the presence of serious, obesity-related comorbidities. The adolescent must have attained or nearly attained physical maturity and have a history of organized attempts at weight management without success. Finally, the adolescent must demonstrate reasonable decision making abilities, be willing to commit to the comprehensive medical and psychological assessment process prior to surgery, and understand and be willing to adhere to the postoperative nutritional guidelines. Readiness and motivation to make long-term dietary and lifestyle changes are major factors when determining if a candidate is appropriate for bariatric surgery. If the patient is struggling to commit to dietary changes required of surgery, it is often recommended that they enter a pre-surgical medical weightmanagement program or seek individual treatment from a Registered Dietitian to help them better prepare for surgery.
The most common surgical procedures include laparoscopic adjustable gastric banding (LAGB) and the Rouxen-Y gastric bypass (RYGB). In both procedures, food intake is limited by the creation of a gastric pouch (approximately $30 \mathrm{ml}$ in size) at the base of the esophagus. RYGB operations have a restrictive component, but also are thought to induce weight loss through selective malabsorption and favorable effects on gut peptides. The stomach and part of the intestine are bypassed by attaching the small pouch to a limb of the intestine, thus, limiting the absorptive surface. Both types of operations are routinely performed laparoscopically.

As has been reviewed in detail elsewhere, adolescent obesity is associated with a number of untoward psychosocial characteristics, including increased symptoms of depression and impaired self-esteem, body image, and quality of life (e.g., Cooperberg and Faith 2004). Not surprisingly, these issues may be even more profound in adolescents with extreme obesity (e.g., Benoit Ratcliff et al. 2011). For example, Kim et al. (2008) found that 22 of 25 (88\%) adolescents received a psychiatric diagnosis based on their psychological evaluation prior to bariatric surgery (which is required by the vast majority of bariatric programs and third party payers throughout the country). Depression was the most common condition, diagnosed in 17 patients $(65 \%)$. Four patients had anxiety disorders, one of whom had concurrent depression. One patient had a diagnosis of schizophrenia. Significant psychological issues, such as uncontrolled depression, substance abuse, thought disorders or significant family discord, typically are considered contraindications to bariatric surgery.

\section{Postoperative Outcomes}

Two years postoperatively, adults typically lose $50-60 \%$ of excess body weight with RYGB procedures and 40-50\% with the LAGB (Buchwald et al. 2004). There are a number of small reports of comparable outcomes in adolescents (e.g., Inge et al. 2004a; Sugerman et al. 2003). Weight loss following bariatric surgery is associated with significant improvements in obesity-related co-morbidities in adults and adolescents. These impressive outcomes must be balanced by the incidence of complications. Early postoperative complications occur in $5-10 \%$ of patients, while late complications have been reported in at least $25 \%$ of patients (Buchwald et al. 2004). Approximately 25\% of patients fail to reach the typical postoperative weight loss or begin to regain large amounts of weight within the first few postoperative years (Sjostrom et al. 2004). Among adolescents, 5 of 33 patients regained some or all of their weight within the first decade after surgery (Sugerman et al. 2003). These suboptimal results typically are 
attributed to poor adherence to the postoperative diet or a return of maladaptive eating behaviors (Sarwer et al. 2005).

Patients must adhere to a rigorous diet following bariatric surgery. In 2008, the American Society of Metabolic and Bariatric Surgery (ASMBS) published the Allied Health Nutritional guidelines for the Surgical Weight Loss Patient (ASMBS 2008). After surgery, patients typically begin with a liquid diet and progress to pureed and soft foods during the first several weeks. At approximately 2 months postoperatively, patients typically return to a diet of regular foods. Common dietary recommendations also include using meal replacement products to control portion sizes, reducing fat and sugar intake, reducing alcohol consumption, increasing consumption of fruits and vegetables, consuming meals consistently throughout the day, and preparing meals at home instead of eating out. A commitment to life-long dietary changes is required of patients undergoing any of the bariatric surgical procedures.

Despite the guidelines, a number of studies have suggested that adherence to the postoperative diet is poor. Caloric intake often increases significantly during the postoperative period (Sarwer et al. 2008). These increases in caloric intake likely contribute to weight regain, which typically begins during the second postoperative year (Sjostrom et al. 2004). While total caloric intake typically increases during the postoperative period, a small subset of bariatric surgery patients suffers from malnutrition. Most cases of malnutrition among bariatric surgery patients appear to be responsive to improved dietary adherence or vitamin supplementation (Xanthakos and Inge 2006). Nevertheless, these problems may be of greater relevance for adolescent patients because of their developmental status at the time of surgery, as well as their longer life span.

\section{Disordered Eating after Bariatric Surgery}

Disordered eating, particularly binge eating, is thought to be relatively common among candidates for bariatric surgery. Binge eating disorder (BED) is characterized by the consumption of an objectively large amount of food in a brief period of time (i.e., $2 \mathrm{~h}$ ) with the patient's report of subjective loss of control during the overeating episode (Spitzer et al. 1992). Patients with BED do not engage in a compensatory behavior, such as vomiting, laxative abuse, or excessive exercise, following the binge episode, which distinguishes BED from bulimia nervosa. Although initial reports suggested that up to $50 \%$ of adult bariatric surgery patients may suffer from the disorder, more recent studies suggest that approximately $5-15 \%$ of patients have the condition (e.g., Allison et al. 2006).
Estimates suggest that less than $3 \%$ of obese adolescents meet criteria BED (Johnson et al. 2002). The prevalence of sub-threshold binge eating among overweight adolescents who present for weight loss treatment, however, is considerably larger (20-30\%) (e.g. Decaluwe et al. 2003; Glasofer et al. 2007; Isnard et al. 2003). A few studies have looked at binge eating behavior in adolescents who presented for bariatric surgery. Zeller et al. (2006) found that $13 \%$ of candidates for bariatric surgery reported symptomatology consistent with a diagnosis of BED, a rate comparable with a comparison group of extremely obese teenagers not interested in surgery $(15 \%)$.

Studies have found that, after bariatric surgery, a significant minority of patients reported feelings of loss of control consistent with BED. In other studies, it appears that binge eating is related to smaller weight losses, or weight regain within the first two postoperative years (e.g., Kalarchian et al. 2002) even though bariatric surgery makes it impossible for them to eat excessively large amounts of food. This may be caused by stretching of the gastric pouch, allowing for increased food intake over time. A recent study, however, suggested no differences in weight loss at 1 year after surgery in persons with and without BED (Wadden et al. 2011).

\section{Psychosocial Outcomes after Bariatric Surgery}

As reviewed in detail elsewhere, numerous studies have found that the majority of adult bariatric patients experience psychological improvements postoperatively (e.g., Sarwer et al. 2005). Most psychosocial characteristics, including self-esteem, depressive symptoms, health-related quality of life, and body image improve dramatically in the first year after surgery. These psychosocial benefits, however, may be limited to the first few postoperative years. The impact of bariatric surgery on longer-term psychological functioning is largely unknown.

Despite the impressive psychological improvements following surgery, there appears to be a small yet significant minority of patients who experience behavioral or psychological complications following bariatric surgery. These complications include depression and suicide, disordered eating, body image dissatisfaction, sexual dysfunction and/or marital discord, and substance abuse (Sarwer and Fabricatore 2010). There is a similar, if not greater, concern for these and other psychological complications in teenagers who undergo bariatric surgery. This population has a high prevalence of psychological dysfunction and disordered eating practices. Detection and treatment of these psychological and behavioral problems may be of particular importance during adolescence. 


\section{Conclusions and Future Directions}

Our country's obesity problem has gone essentially unchecked for the past several decades. A majority of Americans are currently overweight or obese. Many of those individuals are suffering from significant weightrelated health problems which may threaten their life expectancy. Furthermore, the health care costs associated with treating these conditions, on both the individual and societal level, are staggering and represent a significant threat to our country's economic well being over the next several decades.

Although there is much greater awareness of these issues than before, researchers, clinicians and policy makers have made little progress to date. The past decade has witnessed an increased emphasis on prevention, particularly among American youth. Unfortunately, studies of the effectiveness of prevention efforts have not been encouraging (e.g., Foster et al. 2010). On the individual level, behavioral and dietary treatments typically lead to modest weight losses and corresponding improvements in health. Unfortunately, these benefits are rarely maintained beyond the period of active treatment, leaving many patients back where there they started with regard to their weight and health, and discouraged about their ability to reduce and maintain their weight in the future.

This cycle is particularly concerning when we consider the growth of childhood and adolescent obesity. It is very disconcerting to consider the potential psychological toll that obesity can have on a child growing up in a culture that overemphasizes and equates thinness with physical beauty. Add to the mix frustration of repeated weight loss and regain, as well as the societal and economic burden of obesity noted above, and it is hard not to be discouraged when thinking about childhood obesity.

For these as well as other reasons, bariatric surgery has become an increasingly popular treatment option for adults with extreme obesity. The postoperative weight losses are sizable and typically lead to significant improvements in obesity-related comorbidities and psychosocial status. However, a significant minority of patients appears to struggle with these issues, and, as a result, experience suboptimal weight losses or premature and significant weight regain. Thus, there is growing consensus that improvements in dietary intake and eating behaviors are critical to long term success following bariatric surgery.

There has been little study of these issues in adolescents who undergo bariatric surgery. Dietary intake and eating behaviors may be of even greater importance for adolescents who undergo surgery because of their maturational status and longer life expectancy. Over the past several years, the National Institutes of Health has been funding the Teen-Longitudinal Assessment of Bariatric Surgery (Teen-LABS) consortium. The primary objective of the consortium is to investigate the safety and efficacy of bariatric surgery in adolescents (Inge et al. 2004c). Adolescents (19 years old or younger) are being recruited from five sites: Cincinnati Children's Hospital Medical Center, Texas Children's Hospital, Children's Hospital of Alabama, Nationwide Children's Hospital, and University of Pittsburgh Medical Center.

The Teen-LABS consortium also is supporting a number of ancillary studies specifically focused on psychological and behavioral issues. One such study is investigating changes in a variety of psychosocial domains, including depressive symptoms, quality of life, body image, and other issues related to socialization, including participation in high-risk health behaviors that may put these adolescents at increased risk for some of the poor psychosocial outcomes detailed above. Another study is looking at changes in eating behavior and dietary intake after surgery to see if adolescents are able to successfully adhere to the rigorous postoperative diet as they transition into early adulthood. These as well as the other studies in the consortium will provide both medical and mental health professionals with vitally important information about the efficacy of bariatric surgery for adolescents.

In summary, obesity represents one of the Western world's most significant health problems. Rates of obesity among both adults and adolescents have risen dramatically over the past several decades. The most popular weight loss treatments-lifestyle modification and pharmacotherapytypically produce modest weight losses which may improve some weight-related health problems. Unfortunately, most individuals who use these treatments regain their weight within a few years and, as a result, return to being obese. This cycle of weight loss and regain is particularly concerning for adolescents, who may end up living with obesity, and its associated medical and mental health consequences, for decades. This scenario, whether considered from the individual or societal level, is sobering. At the present time, bariatric surgery holds the greatest promise in promoting larger and sustained weight losses which are associated with significant improvements in weight-related comorbidities. Unfortunately, only one percent of adults who are heavy enough for surgery currently receive it; the percentage is even smaller for adolescents. This intersection of circumstances-a growing public health problem, a lack of effectiveness of the most common treatments, and an under-utilization of the most efficacious treatment-will challenge professionals from countless disciplines to work together in novel and creative ways to combat the daunting obesity problem facing youth around the world. 


\section{References}

Allied Health Sciences Section Ad Hoc Nutrition Committee, Aills, L., Blankenship, J., Buffington, C., Furtado, M., \& Parrott, J. (2008). ASMBS Allied health nutritional guidelines for the surgical weight loss patient. Surgery for Obesity and Related Diseases, 4(5 Suppl), S73-S108.

Allison, K. C., Wadden, T. A., Sarwer, D. B., Fabricatore, A. N., Crerand, C. E., Gibbons, L. M., et al. (2006). Night eating syndrome and binge eating disorder among persons seeking bariatric surgery: Prevalence and related features. Obesity (Silver Spring), 14(Suppl 2), 77S-82S.

Benoit Ratcliff, M., Jenkins, T. M., Reiter-Purtill, J., Noll, J. G., \& Zeller, M. H. (2011). Risk-taking behaviors of adolescents with extreme obesity: Normative or not? Pediatrics, 127, 827-834.

Berkowitz, R. I., Wadden, T. A., Tershakovec, A. M., \& Cronquist, J. L. (2003). Behavior therapy and sibutramine for the treatment of adolescent obesity: A randomized controlled trial. Journal of the American Medical Association, 289, 1805-1812.

Berkowitz, R. I., Fujioka, K., Daniels, S. R., Hoppin, A. G., Owens, S., Perry, A. C., et al. (2006). Effects of sibutramine treatment in obese adolescents: a randomized trial. Annals of Internal Medicine, 145, 81-90.

Berkowitz, R. I., Wadden, T. A., Gehrman, C. A., Bishop-Gilyard, C. T., Moore, R. H., Womble, L. G., \& Xanthopoulos, M. S. (2010). Meal replacements in the treatment of adolescent obesity: A randomized controlled trial. Obesity (Silver Spring).

Boutelle, K. N., Cafri, G., \& Crow, S. J. (2011). Parent-only treatment for childhood obesity: A randomized controlled trial. Obesity (Silver Spring), 19(3), 574-580.

Buchwald, H., Avidor, Y., Braunwald, E., Jensen, M. D., Pories, W., Fahrbach, K., et al. (2004). Bariatric surgery: a systematic review and meta-analysis. Journal of the American Medical Association, 292(14), 1724-1737.

Caballero, B. (2004). Obesity prevention in children: opportunities and challenges. International Journal of Obesity, 28, S90-S95.

Cole, T. J., Bellizzi, M. C., Flegal, K. M., \& Dietz, W. H. (2000). Establishing a standard definition for child overweight and obesity worldwide: International survey. British Medical Journal, 320, 1240-1243.

Consensus Development Conference Panel. (1991). Gastrointestinal surgery for severe obesity. Annals of Internal Medicine, 115, 956-961.

Cooperberg, J., \& Faith, M. S. (2004). Treatment of obesity II: Childhood and adolescent obesity. In J. K. Thompson (Ed.), Handbook of eating disorders and obesity (pp. 443-462). New York: Wiley.

Daniels, S. R., Arnett, D. K., Eckel, R. H., Gidding, S., Hayman, S., Kumanyika, S., et al. (2005). Overweight in children and adolescents: pathophysiology, consequences, prevention, and treatment. Circulation, 111, 1999-2012.

Decaluwe, V., Braet, C., \& Fairburn, C. G. (2003). Binge eating in obese children and adolescents. International Journal of Eating Disorders, 33, 78-84.

Dietz, W. H., \& Bellizzi, M. C. (1999). Introduction: The use of body mass index to assess obesity in children. American Journal of Clinical Nutrition, 70, 123S-125S.

Foster, G. D., Sherman, S., Borradaile, K. E., Grundy, K. M., Vander Veur, S. S., Nachmani, J., et al. (2008). A policy-based school intervention to prevent overweight and obesity. Pediatrics, 121(4), e794-e802.

Foster, G. D., Linder, B., Baranowski, T., Cooper, D. M., Goldberg, L., Harrell, J. S., et al. (2010). A school-based intervention for diabetes risk reduction. New England Journal of Medicine, 363(5), 443-453.
Freedman, D. S., Mei, Z., Srinivasan, S. R., Berenson, G. S., \& Dietz, W. H. (2007). Cardiovascular risk factors and excess adiposity among overweight children and adolescents: The Bogalusa heart study. The Journal of Pediatrics, 150, 3-5.

Glasofer, D. R., Tanofsky-Kraff, M., Eddy, K. T., Yanovski, S. Z., Theim, K. R., Mirch, M. C., et al. (2007). Binge eating in overweight treatment-seeking adolescents. Journal of Pediatric Psychology, 32, 95-105.

Gortmaker, S. L., Must, A., Perrin, J. M., Sobol, A. M., \& Dietz, W. H. (1993). Social and economic consequences of overweight in adolescence and young adulthood. New England Journal of Medicine, 329, 1008-1012.

Inge, T. H., Garcia, V., Daniels, S., Langford, L., Kirk, S., Roehrig, H., et al. (2004a). A multidisciplinary approach to the adolescent bariatric surgical patient. Journal of Pediatric Surgery, 39, 442-447.

Inge, T. H., Krebs, N. F., Garcia, V. F., Skelton, J. A., Guice, K. S., Strauss, R. S., et al. (2004b). Bariatric surgery for severely overweight adolescents: concerns and recommendations. Pediatrics, 114, 217-223.

Inge, T. H., Zeller, M. H., Garcia, V. F., \& Daniels, S. R. (2004c). Surgical approach to adolescent obesity. Adolescent Medicine, 15, 429-453.

Isnard, P., Michel, G., Frelut, M. L., Vila, G., Falissard, B., Naja, W., et al. (2003). Binge eating and psychopathology in severely obese adolescents. International Journal of Eating Disorders, $34,235-243$.

Johnson, W. G., Hinkle, L. K., Carr, R. E., Anderson, D. A., Lemmon, C. R., Engler, L. B., et al. (1997). Dietary and exercise interventions for juvenile obesity: Long-term effect of behavioral and public health models. Obesity Research, 5, 257-261.

Johnson, J. G., Cohen, P., Kasen, S., \& Brook, J. S. (2002). Childhood adversities associated with risk for eating disorders or weight problems during adolescence or early adulthood. American Journal of Psychiatry, 159, 394-400.

Kalarchian, M. A., Marcus, M. D., Wilson, G. T., Labouvie, E. W., Brolin, R. E., \& LaMarca, L. B. (2002). Binge eating among gastric bypass patients at long-term follow-up. Obesity Surgery, 12, 270-275.

Kim, R. J., Langer, J. M., Baker, A. W., Filter, D. E., Williams, N. N., \& Sarwer, D. B. (2008). Psychosocial status in adolescents undergoing bariatric surgery. Obesity Surgery, 18(1), 27-33.

Kuczmarski, R. J., Ogden, C. L., Guo, S. S., Grummer-Strawn, L. M., Flegal, K. M., Mei, Z., et al. (2002). [2000] CDC growth charts for the United States: Methods and development. Vital Health Stat, 11(246), 1-190.

Ogden, C. L., Flegal, K. M., Carroll, M. D., \& Johnson, C. L. (2002). Prevalence and trends in overweight among US children and adolescents 1999-2000. Journal of the American Medical Association, 288, 1728-1732.

Ogden, C. L., Carroll, M. D., Curtin, L. R., Lamb, M. M., \& Flegal, K. M. (2010). Prevalence of high body mass index in US children and adolescents, 2007-2008. Journal of the American Medical Association, 303(3), 242-249.

Oude Luttikhuis, H., Baur, L., Jansen, H., Shrewsbury, V. A., O’Malley, C., \& Summerbell, C. D. (2009). Interventions for treating obesity in children. Cochrane Database of Systematic Reviews, 1, CD001872.

Robinson, T. N. (1999). Behavioural treatment of childhood and adolescent obesity. International Journal of Obesity and Related Metabolic Disorders, 23(Suppl 2), S52-S57.

Robinson, T. N., \& Sirard, J. R. (2005). Preventing childhood obesity. American Journal of Preventative Medicine, 28(2S2), 194-201.

Santry, H. P., Gillen, D. L., \& Lauderdale, D. S. (2005). Trends in bariatric surgical procedures. Journal of the American Medical Asociation, 294(15), 1909-1917. 
Sarwer, D. B., Wadden, T. A., \& Fabricatore, A. N. (2005). Psychosocial and behavioral aspects of bariatric surgery. Obesity Research, 13, 639-648.

Sarwer, D. B., Fabricatore, A. N., Eisenberg, M. H., Sywulak, L. A., \& Wadden, T. A. (2008). Self-reported stigmatization among candidates for bariatric surgery. Obesity (Silver Spring), 16(Suppl 2), S75-S79.

Sarwer, D. B., \& Fabricatore, A. N. (2010). Psychological issues before and after bariatric surgery. In: M. Dietl, M. Gagner, J. Dixon, J. Himpens, \& A. Madan (Eds.), Handbook of obesity surgery. Canada: FD-Communications.

Sjostrom, L., Lindroos, A. K., Peltonen, M., Torgerson, J., Bouchard, C., Carlsson, B., et al. (2004). Lifestyle, diabetes, and cardiovascular risk factors 10 years after bariatric surgery. New England Journal of Medicine, 351, 2683-2693.

Speiser, P. W., Rudolf, M. C., Anhalt, H., Camacho-Hubner, C., Chiarelli, F., et al. (2005). Consensus statement: Childhood obesity. The Journal of Clinical Endocrinology and Metabolism, 90, 1871-1887.

Spitzer, R. L., Devlin, M., Walsh, B. T., Hasin, D., Wing, R. R., Marcus, M., et al. (1992). Binge-eating disorder: A multi site field trial of the diagnostic criteria. International Journal of Eating Disorders, 3, 191-203.

Sugerman, H. J., Sugerman, E. L., DeMaria, E. J., Kellum, J. M., Kennedy, C., Mowry, Y., et al. (2003). Bariatric surgery for severely obese adolescents. Journal of Gastrointestinal Surgery, 7, 102-107.

Tsai, W. S., Inge, T. H., \& Randall, S. B. (2007). Bariatric surgery in adolescents: recent national trends in use and in-hospital outcome. Archives of Pediatric and Adolescent Medicine, 161, 219-221.

van Dam, R. M., Willett, W. C., Manson, J. E., \& Hu, F. B. (2006). The relationship between overweight in adolescence and premature death in women. Annals of Internal Medicine, 145, 91-97.
Wadden, T. A., Faulconbridge, L. F., Jones-Corneille, L. R., Sarwer, D. B., Fabricatore, A. N., Thomas, J. G., \& Williams, N. N. (2011). Binge eating disorder and the outcome of bariatric surgery at one year: A prospective, observational study. Obesity (Silver Spring), (Epub ahead of print).

Whitaker, R. C., Wright, J. A., Pepe, M. S., Seidel, K. D., \& Dietz, W. H. (1997). Predicting obesity in young adulthood from childhood and parental obesity. New England Journal of Medicine, 337, 869-873.

Xanthakos, S. A., \& Inge, T. H. (2006). Nutritional consequences of bariatric surgery. Current Opinion in Clinical Nutrition and Metabolic Care, 9, 489-496.

Zeller, M. H., Roehrig, H. R., Modi, A. C., Daniels, S. R., \& Inge, T. H. (2006). Health-related quality of life and depressive symptoms in adolescents with extreme obesity presenting for bariatric surgery. Pediatrics, 117, 1155-1161.

\section{Author Biographies}

David B. Sarwer is Associate Professor of Psychology in Psychiatry and Surgery at the University of Pennsylvania School of Medicine as well as Director of Clinical Services at the Center for Weight and Eating Disorders. He received his doctorate in clinical psychology from Loyola University Chicago. His major research interests include the psychosocial and behavioral aspects of obesity.

Rebecca J. Dilks is a Research Coordinator at the Center for Weight and Eating Disorders. A Registered Dietitian and a Licensed Dietitian-Nutritionist, Ms. Dilks received a B.S. from Immaculata University. Her major research interests focus on the nutritional management of obesity. 